\title{
Leftover opioids following adult surgical procedures: a systematic review and meta- analysis
}

Lori Schirle ${ }^{1 *}$ D, Amanda L. Stone ${ }^{2}$, Matthew C. Morris ${ }^{3}$, Sarah S. Osmundson ${ }^{4}$, Philip D. Walker ${ }^{5}$, Mary S. Dietrich ${ }^{1,6}$ and Stephen Bruehl ${ }^{2}$

\begin{abstract}
Background: US opioid prescribing and use escalated over the last two decades, with parallel increases in opioid misuse, opioid-related deaths, and concerns about diversion. Postoperatively prescribed opioids contribute to these problems. Policy makers have addressed this issue by limiting postoperative opioid prescribing. However, until recently, little data existed to guide prescribers on opioid needs postoperatively. This meta-analysis quantitatively integrated the growing literature regarding extent of opioids leftover after surgery and identified factors associated with leftover opioid proportions.

Methods: We conducted a meta-analysis of observational studies quantifying postoperative opioid consumption in North American adults, and evaluated effect size moderators using robust variance estimation meta-regression. Medline, EMBASE, Cumulative Index of Nursing and Allied Health Literature, and Cochrane Database of Systematic Reviews were searched for relevant articles published January 1, 2000 to November 10, 2018. The Methodological Index for Non-Randomized Studies (MINORS) tool assessed risk of study bias. The proportion effect size quantified the primary outcome: proportion of prescribed postoperative opioids leftover at the time of follow-up. Primary meta-regression analyses tested surgical type, amount of opioids prescribed, and study publication year as possible moderators. Secondary meta-regression models included surgical invasiveness, age, race, gender, postoperative day of data collection, and preoperative opioid use.
\end{abstract}

Results: We screened 911 citations and included 44 studies (13,068 patients). The mean weighted effect size for proportion of postoperative opioid prescriptions leftover was $61 \%$ (95\% Cl, 56-67\%). Meta-regression models revealed type of surgical procedure and level of invasiveness had a statistically significant effect on proportion of opioids leftover. Proportion of opioids leftover was greater for "other soft tissue" surgeries than abdominal/pelvic surgeries, but did not differ significantly between orthopedic and abdominal/pelvic surgeries. Minimally invasive compared to open surgeries resulted in a greater proportion of opioids leftover. Limitations include predominance of studies from academic settings, inconsistent reporting of confounders, and a possible publication bias toward studies reporting smaller leftover opioid proportions.

Conclusions and implications of key findings: A significant proportion of opioids are leftover postoperatively. Surgery type and level of invasiveness affect postoperative opioid consumption. Integration of such factors into prescribing guidelines may help minimize opioid overprescribing while adequately meeting analgesic needs.

Keywords: Postoperative pain, Postoperative care, Opioid usage, Acute pain, Pain medications, Outpatient

\footnotetext{
*Correspondence: lori.schirle@vanderbilt.edu

${ }^{1}$ School of Nursing, Vanderbilt University, 461 21st Avenue South, Nashville,

TN 37240, USA

Full list of author information is available at the end of the article
}

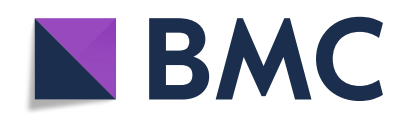

(c) The Author(s). 2020 Open Access This article is licensed under a Creative Commons Attribution 4.0 International License, which permits use, sharing, adaptation, distribution and reproduction in any medium or format, as long as you give appropriate credit to the original author(s) and the source, provide a link to the Creative Commons licence, and indicate if changes were made. The images or other third party material in this article are included in the article's Creative Commons licence, unless indicated otherwise in a credit line to the material. If material is not included in the article's Creative Commons licence and your intended use is not permitted by statutory regulation or exceeds the permitted use, you will need to obtain permission directly from the copyright holder. To view a copy of this licence, visit http://creativecommons.org/licenses/by/4.0/ The Creative Commons Public Domain Dedication waiver (http://creativecommons.org/publicdomain/zero/1.0/) applies to the data made available in this article, unless otherwise stated in a credit line to the data. 


\section{Background}

Despite increasing national attention and concentrated policy efforts, the opioid epidemic continues to grow, claiming 130 lives daily and contributing to an unprecedented recent decrease in life expectancy in the USA (US) [1]. Opioid analgesics are commonly prescribed for acute pain following surgical interventions [2], with over one million surgical procedures performed annually in the US [3]. Wide variations in opioid prescribing across providers and in opioid consumption across patients can result in a significant proportion of leftover opioids following surgery $[4,5,6]$. For example, one study reported discharge opioid prescriptions ranging from zero to 100 pills after laparoscopic cholecystectomy, while patients consumed on average less than 10 pills [5]. Given that more than half of individuals who misuse prescription opioids obtain them from a friend or relative's supply [7], leftover opioids following surgery represent a significant public health issue. Beyond diversion concerns, larger quantities of opioids prescribed following surgery have been associated with increased opioid consumption $[6,8,9]$. In turn, evidence suggests that greater postsurgical opioid consumption may contribute to long-term opioid use $[10,11]$ and development of opioid use disorders [12].

Recent policy initiatives enacted by states, insurers, and pharmacies have sought to decrease opioid diversion and misuse by placing limits on opioid quantities prescribed after surgical procedures [13]. Although these efforts may decrease the absolute number of opioids leftover by patients, considerable quantities of opioids may nonetheless remain unused in patients who consume few to no opioids after surgery. Conversely, these policies may cause unintended harm to patients requiring larger amounts of opioids for adequate pain control, as poorly managed postoperative pain is a major risk factor for developing chronic postsurgical pain [14].

One reason cited for variation in opioid prescribing practices is the lack of adequate data-driven knowledge about analgesic needs after surgery to guide clinician opioid prescribing [15]. This knowledge gap is particularly important to address given the opioid prescribing policy changes currently being enacted. Over the past several years, a growing number of studies have evaluated surgery-specific opioid consumption patterns, although these data have yet to be integrated quantitatively. A 2017 qualitative systematic review summarized six studies addressing postoperative opioid consumption, and reported that $42-71 \%$ of prescribed opioids remain unused, with most stored in unsecure locations [16]. A second qualitative review published several months later identified 11 studies addressing postoperative opioid consumption, and reported similar findings [17]. The aim of the current meta-analysis is to quantitatively integrate for the first time the rapidly growing literature regarding extent of leftover opioids after surgery and identify factors associated with the amount of leftover opioids. A primary meta-regression model evaluated factors that may be linked to extent of leftover opioids following surgery, including surgical type, amount prescribed, measurement method, changes in prescribing patterns over time, and geographic region. Secondary meta-regression models also evaluated the influence of demographic variables, surgical invasiveness, use of opioids at the time of surgery, and timing of postoperative opioid consumption data collection. Based upon previous research, our primary hypothesis was that a substantial percentage of opioids prescribed would be leftover $[16,17]$, and that orthopedic surgeries would result in fewer leftover opioids due to higher pain intensity related to the greater bone/soft tissue disruption involved $[18,19]$.

\section{Methods}

This meta-analysis was conducted according to the Preferred Reporting Items for Systematic Review and MetaAnalysis (PRISMA) guidelines (Fig. 1). The PRISMA checklist can be found in supplemental documentation [20]. No prior protocol was published for this project.

\section{Eligibility criteria}

We included studies in adult surgical populations of any design published in North America that reported both the amount of opioids prescribed and consumed for the postsurgical period after patient discharge. We limited our search to North America, as beliefs about opioid prescribing and pain control expectations vary between countries and regions [21], and the US and Canada are the top opioid consuming nations, with similar pharmaceutical industry influences [22]. Exclusion criteria for the current meta-analysis were (a) significant presence of pediatric patients ( $>5 \%$ of study population), (b) only reported inpatient opioid consumption, (c) did not quantify opioid prescriptions and consumption by morphine milligram equivalents (MME) or number of pills, and (d) use of an intervention that would affect opioid consumption patterns, as interventions would obscure the natural variations in opioid use that were the focus of this review (no-intervention control conditions in studies testing an intervention were included when available).

\section{Data sources}

Medline (via PubMed), EMBASE (OvidSP), Cumulative Index of Nursing and Allied Health Literature (CINAHL) (EBSCOhost), and the Cochrane Database of Systematic Reviews (Wiley) were searched for relevant 


\section{PRISMA Flow Diagram}
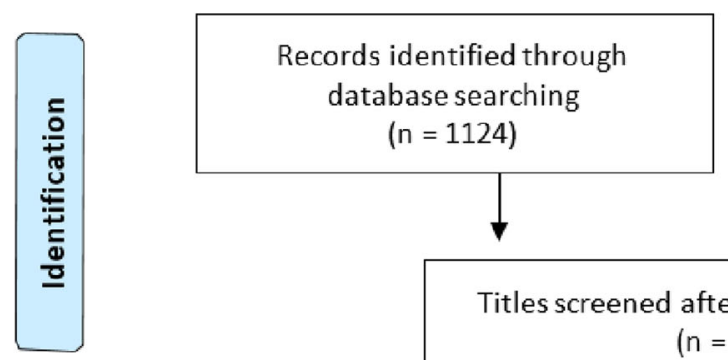

Records identified through eligible article reference lists

$(n=1124)$

$(\mathrm{n}=3)$
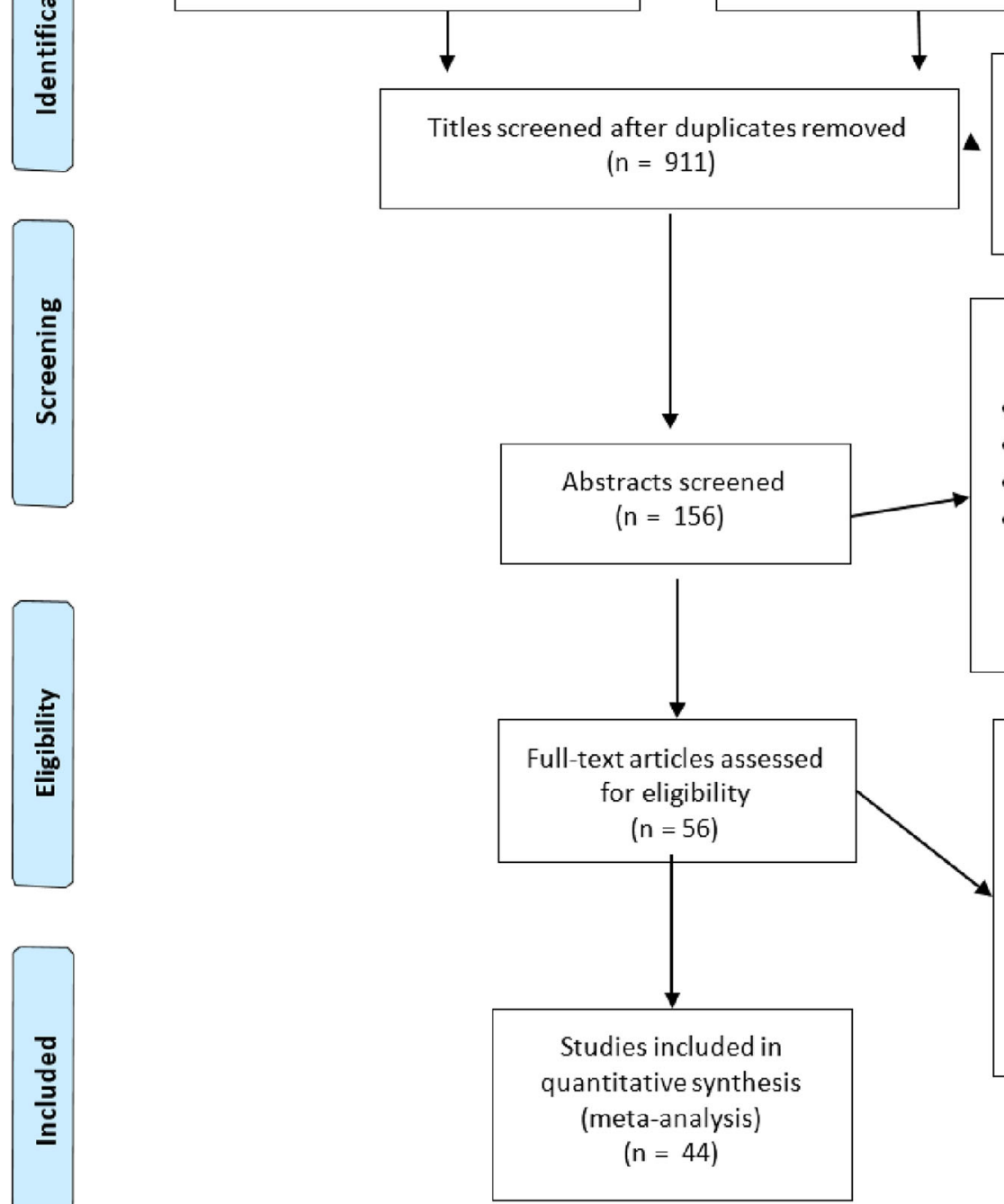

Records excluded $(n=755)$

- Non-surgical population, pediatric population, non-empirical literature

Records excluded

$(n=100)$

- Non-surgical $(n=4)$

- Pediatric population $(n=5)$

- Non-empirical ( $n=16)$

- Did not measure opioid consumption postdischarge $(n=75)$

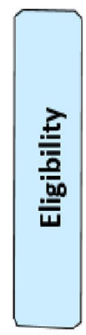

Full-text articles excluded

$$
(n=12)
$$

- Did not quantify opioid consumption post-

discharge $(n=11)$

- Intervention to change opioid prescribing with no pre-test or control $(n=1)$

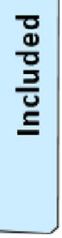

Studies included in

uantitative synthesis

$$
(n=44)
$$

Fig. 1 PRISMA flow diagram

articles published from January 1, 2000 to November 10, 2018. The final search was performed on December 10, 2018. Online biomedical literature databases were searched by using a combination of keywords and database-specific subject headings determined by a biomedical librarian (P.W.) who has expertise in biomedical literature searches. The search strategy is available in supplemental documentation. Reference lists from eligible studies and prior review articles on the topic were scanned for other eligible studies that may have been missed by search criteria.

\section{Data extraction and quality assessment}

Two authors (A.S. and L.S.) independently extracted relevant study data using a data extraction template. Extracted data included surgery type (abdominal/pelvic, orthopedic, other soft tissue), invasiveness (minimally invasive, open), sample demographic characteristics (sex, 
race, age), geographic region based upon US Censusdesignated region $\mathrm{s}$ [23] (1, Northeast; 2, Midwest; 3, South; 4, West; 5, Multiple Regions; 6, Canada) of data collection, year of publication, aggregate amount of opioid prescribed (converted to morphine milligram equivalents-MME [24]) and consumed per surgical type, timing of opioid consumption data collection relative to surgery, preoperative opioid use, and type of summary statistic for prescribed and consumed opioids reported (mean or median). These factors were chosen based on a preliminary review of eligible studies and the data available as well as hypothesized factors which could affect opioid consumption patterns. Attempts were made to contact authors of selected studies to supply key missing data (supplementary documentation). Given the observational nature of all studies included in this meta-analysis (Level II and III evidence only), two authors (A.S. and L.S.) independently provided a detailed assessment of the quality of studies using the Methodological Index for Non-Randomized Studies (MINORS) risk of bias tool [25] and demonstrated adequate agreement (87.2\%). Disagreements on data extraction or quality ratings were resolved by discussion and consensus or consultation of a third author (M.D.).

\section{Data analytic plan}

Statistical analyses were conducted in the $\mathrm{R}$ statistical environment (version 3.6.1). The proportion effect size $\left(E S_{P}\right)$ was used to determine the proportion of postsurgical opioids prescribed that was leftover at the time of follow-up. Proportions were computed as the amount of prescribed opioids (mean/median number of pills/ MMEs) remaining at post-surgery assessment (numerator) divided by the amount of opioids (mean/median number of pills/MMEs) that were originally prescribed (denominator). Effect size means and variances were estimated from studies reporting medians using established methods [26]. Due to the approximately normal distribution of observed proportions, no transformation of the distribution of $E S_{P}$ was required [27]. An innovative technique known as robust variance estimation (RVE) meta-regression was used to handle statistically dependent effect sizes (i.e., multiple effect sizes nested within studies) [28]; RVE was implemented in R using the robumeta [29] and clubSandwich [30] packages. RVE analyses included small sample adjustments for $t$ tests. Two-level mixed effects models were specified to allow simultaneous estimation of within-study (level 1) and between-study (level 2) parameters. The intra-class correlation used to calculate variance components in the random effects model $(\rho)$ was set at 0.8 . The proportion of observed variation across studies that is due to true effects-rather than sampling error-was assessed with the $I^{2}$ statistic [31, 32]. High $I^{2}$ values (defined as greater than or equal to $75 \%$ [33]) suggest that the proportion of opioids leftover likely depends on moderators. The $I^{2}$ statistic is a relative measure and does not reflect the absolute amount of heterogeneity [33]. To better capture dispersion, effect sizes are reported with $95 \%$ confidence intervals.

RVE meta-regression models tested effect size moderators. All studies reviewed had data available for the primary moderators of interest: surgical type (categorical: abdominal/pelvic, other soft tissue, and orthopedic), amount of opioids prescribed (in MMEs), and study publication year. To address methodological variability and maximize generalizability of results, the metaregression models statistically controlled for effect size measurement method (mean versus median), publication year, and for the geographic region in which data were obtained. Secondary meta-regression models were conducted for the following potential moderators given their availability in only a subset of studies: surgical invasiveness (open vs. minimally-invasive), age, race, gender, postoperative day of data collection, and preoperative opioid use. All models were evaluated for possible multicollinearity, and no issues were noted. A type I error rate of 0.05 was used for assessing statistical significance (i.e., $p<0.05)$.

\section{Results}

\section{Study selection and characteristics}

The initial search criteria identified 911 unique citations (Fig. 1) [20]. After an initial screening of titles and abstracts, 156 full-text articles were assessed for eligibility. Of these articles, 44 studies met eligibility criteria and were included in the final analysis (Table 1). Publication dates ranged from 2004-2018 with > 80\% published in 2017 or later. Studies represented a broad variety of surgeries ranging from those with minimal tissue disruption (e.g., carpal tunnel repair) to those with major bone and tissue disruption (e.g., spinal fusion). The majority of studies were conducted at single academic medical centers in the Eastern US on predominately Caucasian populations and used a prospective observational cohort design with moderate risk of bias (Table 1). Most studies obtained opioid prescription data through electronic health record review or patient report, and opioid consumption data through patient report via phone, electronic, or in-person survey. A small number of studies used observational methods (e.g., pill count) to reduce self-report bias. Studies varied in their methods for reporting (i.e., number of pills vs. MMEs) and summarizing (i.e., mean vs. median) opioid consumption (Table 1). Of the 44 studies included, 3 were deemed at high risk of bias, 29 were deemed at medium risk of bias, and the remaining 12 studies were deemed at low risk of bias. The primary risks of bias detected were lack of an a priori power 


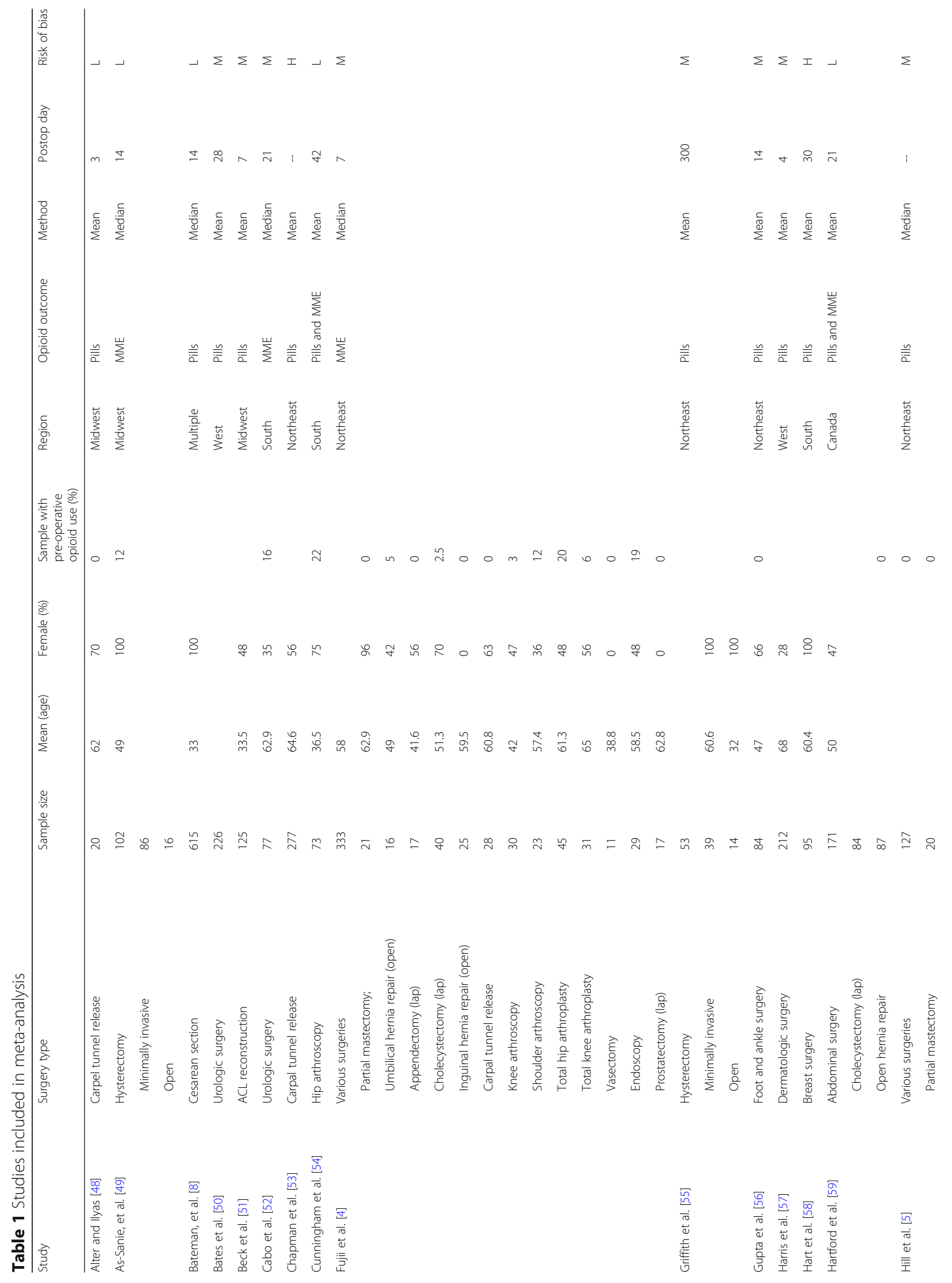




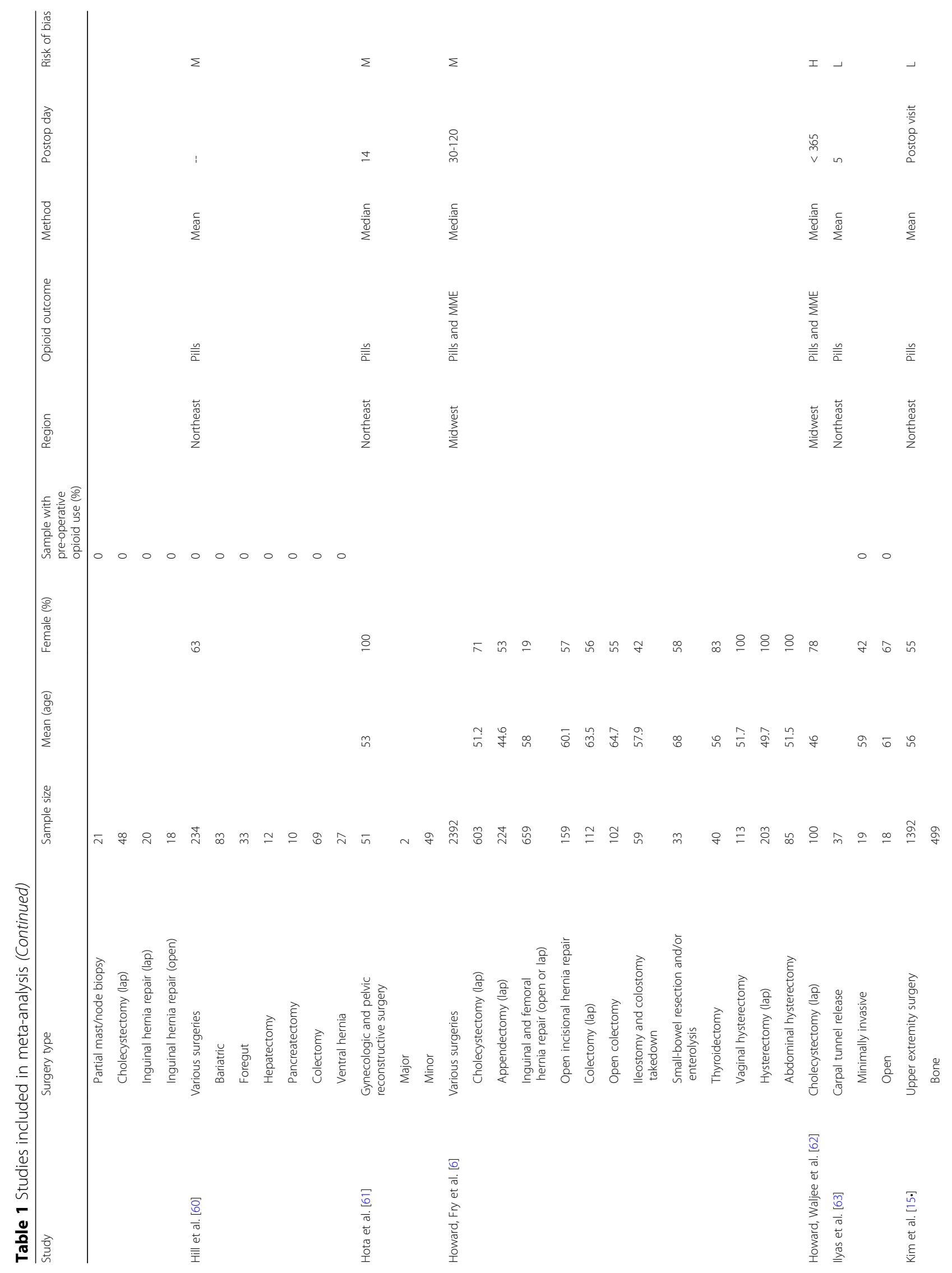




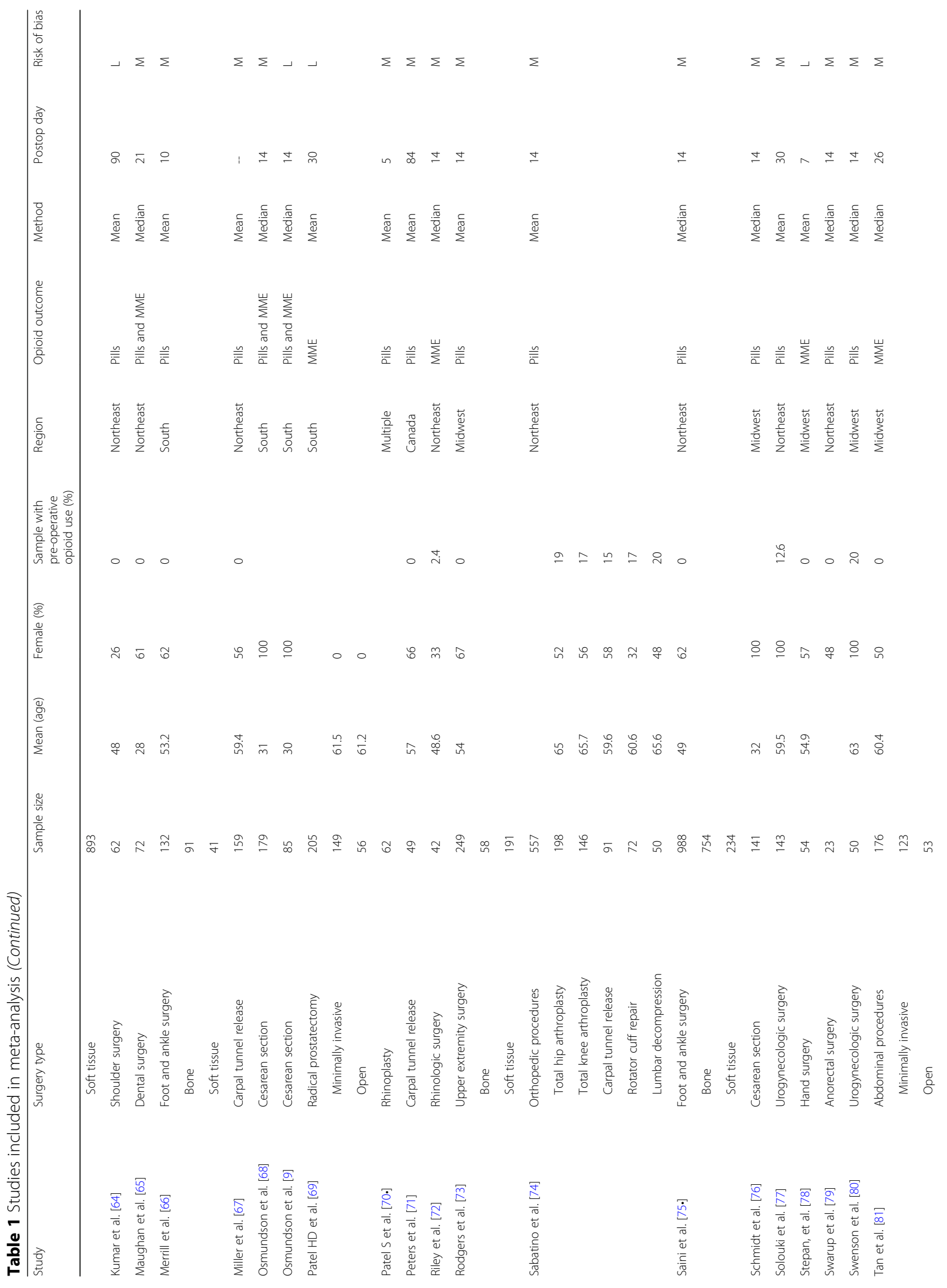




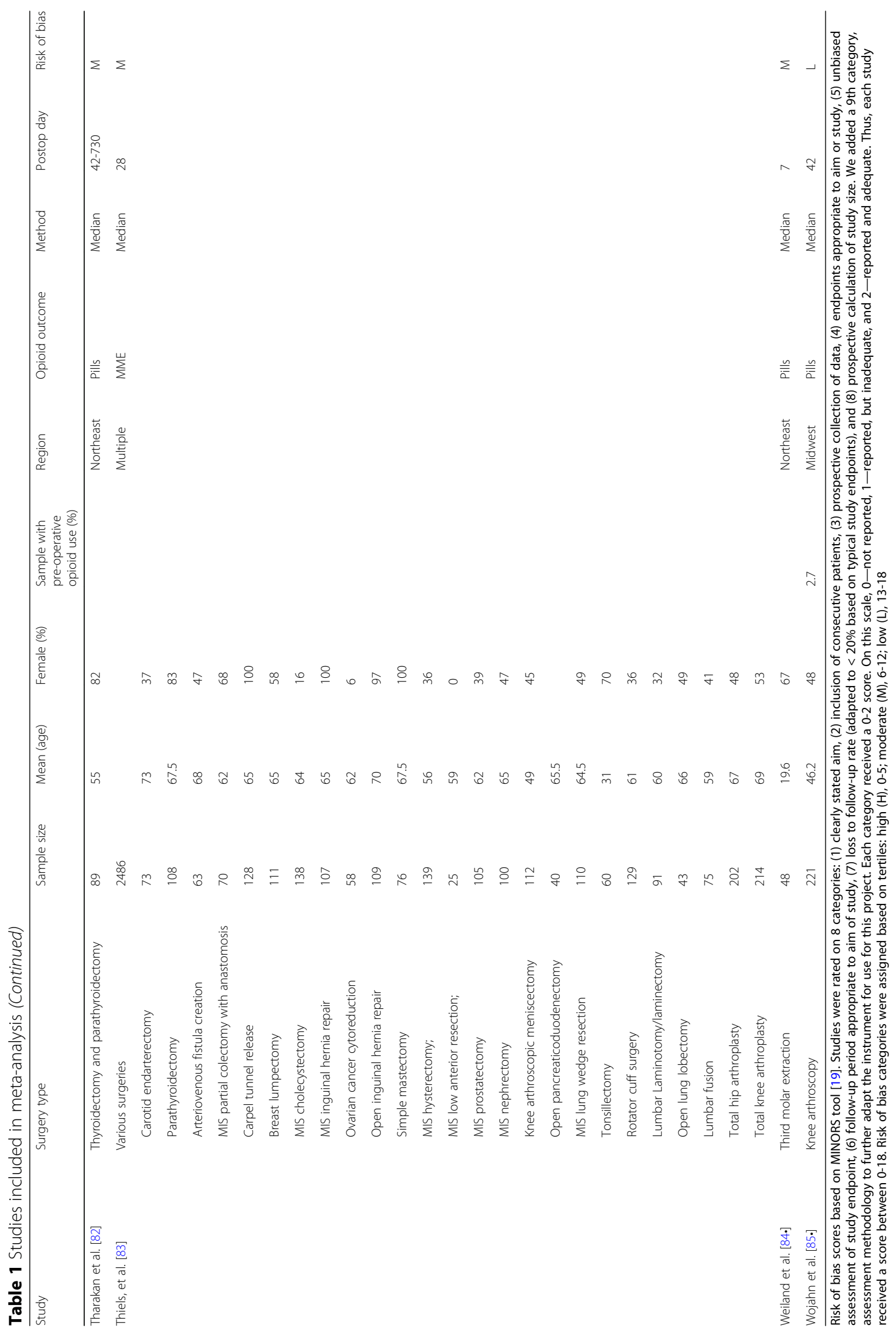


calculation to determine sample size, presence of bias for the endpoint (e.g., study conducted by authors who were primary prescribing physicians in the study), and low response rates to follow-up surveys. RVE meta-regression did not reveal a significant overall effect of study bias risk ratings ( 1 , low risk; 2 , medium risk; 3 , high risk) on proportion of prescribed opioids leftover $(b=0.04, S E=0.05$, $p=0.42,95 \% \mathrm{CI},-0.06,0.15)$.

\section{Primary analyses}

Across 115 effect sizes drawn from 44 studies $(n=13$, 068 patients), the mean weighted effect size for the proportion of prescribed postsurgical opioids leftover at follow-up was $61 \%(t=23.1, d f=42.5,95 \% \mathrm{CI}, 56-67 \%)$. The summary statistics reported in the included studies were weighted by sample size and pooled to estimate that 2,909,744 prescribed MMEs were represented in those studies (i.e., equivalent of 581,949 $5 \mathrm{mg}$ hydrocodone tablets). Thus, results across the included studies indicate that a total equivalent of $354,9895 \mathrm{mg}$ hydrocodone tablets were leftover, or 27 hydrocodone tablets prescribed, but not used per person. The $I^{2}$ value of 94.9\% reveals variation of true effects (as opposed to sampling error) and indicates that the mean weighted effect size for the proportion of prescribed postsurgical opioids leftover may not be the most appropriate estimate for all studies. Instead, this $I^{2}$ value suggests that the proportion of opioids leftover likely depends on potential moderators, which supports the subsequent use of RVE meta-regression.

Primary RVE meta-regression models revealed a significant overall moderating effect of surgical type on the proportion of opioid prescriptions leftover $(b=0.09, S E$ $=0.03, p<0.01,95 \%$ CI, 0.03, 0.15) (Table 2). This model accounted for $34 \%$ of the between-study variance. As shown in Fig. 2, specific surgical type contrasts indicated that most of that overall effect was explained by significantly greater leftover opioids for "other soft tissue" surgeries (i.e., chest/breast, head/dental, other soft tissue) than for abdominal/pelvic surgeries $(b=0.18, S E$
$=0.05, p<0.01 ; 95 \%$ CI, 0.07, 0.29); proportions did not differ significantly between orthopedic and abdominal/ pelvic surgeries $(b=0.07, S E=0.07, p=0.36 ; 95 \% \mathrm{CI}$, $-0.08,0.22)$, nor between "other soft" and orthopedic ( $b$ $=0.11, S E=0.07, p=0.11 ; 95 \% \mathrm{CI},-0.03,0.25)$. Orthopedic studies reported significantly more variability in postoperative opioids consumed (coefficient of variation $[\mathrm{CV}]=0.37,95 \% \mathrm{CI}, 0.26,0.48$ ) versus abdominal/pelvic $(\mathrm{CV}=0.28,95 \% \mathrm{CI}, 0.23,0.33)$ or other soft tissue $(\mathrm{CV}$ $=0.23,95 \% \mathrm{CI}, 0.17,0.29$ ) (both $p$ values $<0.05$ ) .

Primary RVE meta-regression models did not reveal significant overall effects of measurement method $(b=$ $0.08, S E=0.05, p=0.14,95 \% \mathrm{CI},-0.03,0.19)$, geographic region $(b=-0.01, S E=0.01, p=0.65,95 \% \mathrm{CI}$, $-0.04,0.02)$, publication year $(b=0.02, S E=0.01, p=$ $0.11,95 \% \mathrm{CI},-0.01,0.04)$, or amount of opioids prescribed $(b=-0.0003, S E=0.0002, p=0.15,95 \% \mathrm{CI}$, $-0.0009,0.0002)$. A funnel plot displaying the association between effect size estimates and their standard errors is presented in Fig. 3. Egger's test was significant $(z=$ $-10.23, p<0.001$ ), indicating funnel plot asymmetry. Visual inspection of this funnel plot revealed a potential publication bias toward studies reporting smaller proportions of opioid prescriptions leftover. Examining separate funnel plots for studies reporting means $(n=76)$ versus medians ( $n=39$, see Supplemental Figure 1) indicated an absence of asymmetry for the means-only plot $(z=-0.47, p=0.63)$ but continuing asymmetry for the medians-only plot $(\mathrm{z}=-7.96, p<0.001)$. The latter result revealed a trend for larger medians-only studies to report greater proportions of opioids leftover.

\section{Secondary analyses}

Effects of other moderators on the proportion of prescribed opioids leftover following surgery were assessed for the subset of studies that included each. These models all controlled for measurement method, region, publication year, amount of opioids prescribed, and surgery type (i.e., all variables included in the primary analyses). Findings indicated that more invasive open

Table 2 Summary of primary robust variance estimation meta-regression model predicting proportions leftover of postsurgical opioid prescriptions

\begin{tabular}{lllllll}
\hline & $b$ & $S E$ & $t$ value & df & $95 \% \mathrm{Cl}$ & $p$ \\
\hline Measurement method & 0.082 & 0.053 & 1.54 & 24.33 & $-0.028,+0.192$ & 0.137 \\
Region & -0.006 & 0.014 & 0.47 & 10.50 & $-0.037,+0.024$ & 0.648 \\
Publication year & 0.016 & 0.007 & 2.47 & 2.51 & $-0.007,+0.040$ \\
Opioids prescribed (MME) & -0.0003 & 0.0002 & 1.65 & 6.40 & $-0.0009,+0.0002$ & 0.106 \\
Surgical type & 0.089 & 0.027 & 3.26 & 23.18 & $+0.033,+0.146$ & 0.003 \\
\hline
\end{tabular}

Results were based on 115 effect sizes drawn from 44 studies. The intraclass correlation $(\rho)$ was set at 0.8

Measurement method (mean, 0; median, 1); region (1, Northeast; 2, Midwest; 3, South; 4, West; 5, Multiple regions; 6, Canada); publication year (centered such that 0 , first published study in 2004); surgical type (1, "abdominal/pelvic;" 2 , "orthopedic" [including joint/spine, other boney]; 3 , "other soft tissue" [including chest/ breast, head (dental), other soft tissue])

$b$ unstandardized regression coefficient; SE standard error; $\mathrm{Cl}$ confidence interval 


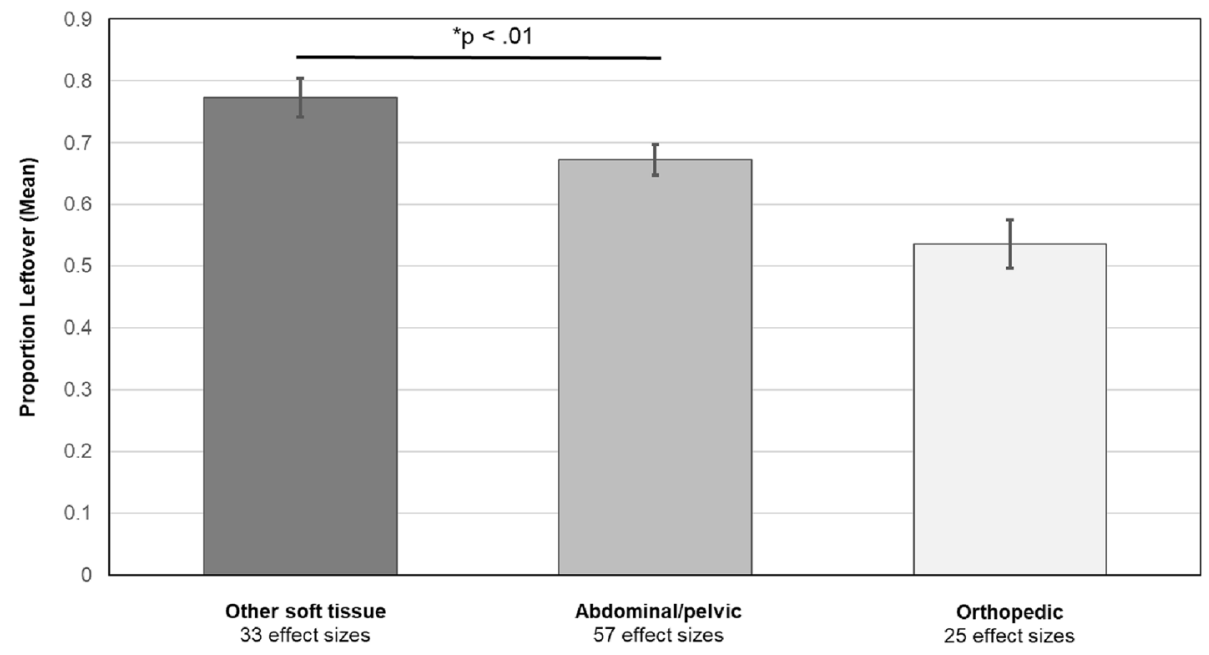

Fig. 2 Mean proportions of postsurgical opioid prescriptions leftover (+/-standard error of the mean) by surgical type

surgical procedures were associated with a significantly lower proportion of prescribed opioids leftover following surgery relative to minimally invasive procedures $(k=36$ studies, number of effect sizes $=87 ; b=-0.16, S E=$ $0.05, p<0.01,95 \% \mathrm{CI},-0.25,-0.06$; Fig. 4). None of the following associations were significant: percentage of the sample that was female $(k=42$ studies, number of effect sizes $=109, b=-0.18, S E=0.10, p=0.10,95 \% \mathrm{CI}$, $-0.39,0.03)$; mean age of sample $(k=40$ studies, number of effect sizes $=102, b=0.003, S E=0.002, p=0.10,95 \%$ CI, $-0.001,0.008)$; percentage of the sample that was Caucasian $(k=19$ studies, number of effect sizes $=51, b$ $=0.21, S E=0.17, p=0.26,95 \% \mathrm{CI},-0.21,0.63)$; postoperative day of data collection (number of days after surgery opioid consumption data was collected) $(k=37$ studies, number of effect sizes $=97, b=0.0002, S E=$ $0.0004, p=0.59,95 \% \mathrm{CI},-0.002,0.002)$; or percentage

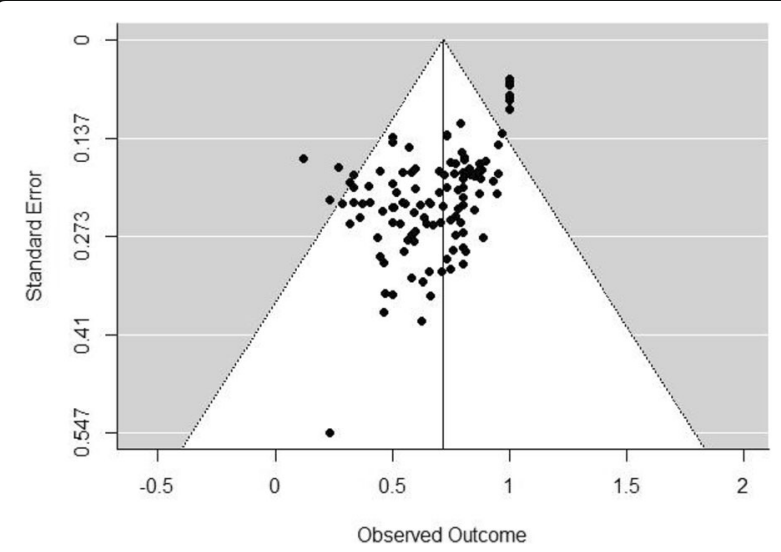

Fig. 3 Funnel plot for studies reporting proportions of postsurgical opioid prescriptions leftover of the sample using opioids preoperatively $(k=26$ studies, number of effect sizes $=57, b=-0.28, S E=0.53, p$ $=0.61,95 \% \mathrm{CI},-1.46,0.91)$.

\section{Discussion}

This meta-analysis synthesized data from 44 studies to quantify the extent of leftover opioids following surgery and evaluate factors potentially associated with the proportion of opioids leftover. Overall, we found that $61 \%$ of opioids prescribed following surgery were leftover, which amounted to approximately $275 \mathrm{mg}$ hydrocodone tablets per person. Assuming a maximum dose of six hydrocodone tablets per day, the average individual was left with enough medication to treat pain for 4.5 additional days. Findings revealed two key moderators associated with proportion of opioids leftover that can guide providers caring for patients after surgery: type of surgery and the degree of invasiveness.

Studies involving surgeries on non-visceral organs (i.e., mastectomy, thyroidectomy) reported significantly more leftover opioids than abdominal/pelvic surgeries, and as expected, minimally invasive techniques were associated with a greater proportion of opioids leftover. Regulatory changes designed to decrease opioid prescribing in these procedures align in part with opioid consumption data from this analysis. For example, Tennessee law limits opioid prescriptions to less than 20 days, depending upon surgery invasiveness [34]. However, patients undergoing abdominal/pelvic surgeries demonstrated fewer leftover opioids relative to other soft tissue procedures (suggesting greater opioid requirements for pain control in abdominal pelvic surgeries). This lack of uniformity across specific soft tissue surgery subtypes is not adequately addressed in the Tennessee prescribing law, potentially contributing to variability in adequacy of pain 


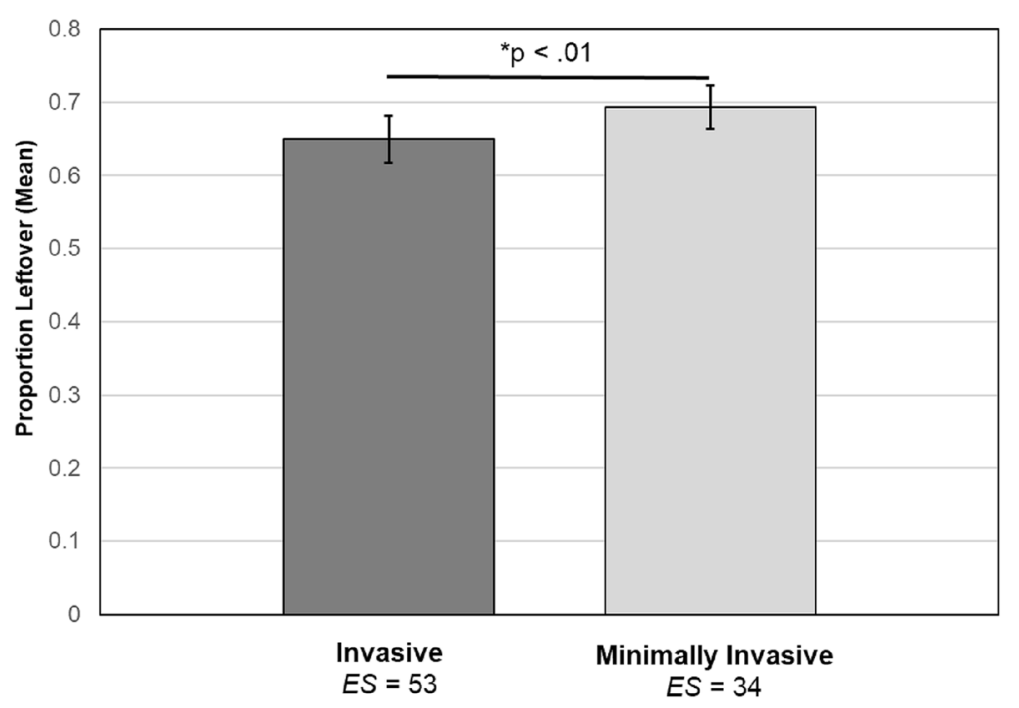

Fig. 4 Mean proportions of postsurgical opioid prescriptions leftover (+/-standard error of the mean) for studies reporting invasive versus minimally invasive surgical procedures

management. Patients undergoing orthopedic surgeries demonstrated the largest variability in opioid consumption relative to abdominal/pelvic or other soft tissue, potentially leaving these patients more vulnerable to blanket opioid restriction policies. These data highlight the potential harm that could occur with policies that uniformly limit opioid prescribing, and the need to engage broad expertise across specialties in developing opioid prescribing guidelines that are supported by specialty-specific data on opioid consumption. Individual variability noted in opioid use particularly among patients undergoing orthopedic surgeries also highlights the potential value of applying a precision medicine approach to opioid prescribing [35], although data to support this approach are still evolving.

The present study adopted a sophisticated RVE megaregression method that can handle complex data structures with dependent effect sizes and that applies an adjustment for small sample bias [32]. Nevertheless, these findings should be interpreted with caution in light of potential publication bias (favoring studies reporting smaller proportions of opioids leftover) that was identified based on funnel plot asymmetry. Follow-up analyses indicated that this asymmetry was driven by studies reporting medians which also tended to be the studies with larger sample sizes. Median values would not be influenced by highly skewed distributions. Means being used particularly with small negatively skewed samples would dramatically under-estimate proportions of opioids leftover. Although RVE meta-regression analyses revealed there were no statistically significant differences between studies reporting means compared to medians, the lack of statistically significant differences cannot be interpreted as similarity or equivalence. Standardizing the reporting of opioid consumption will aid further meta-analytic efforts. Based on our review of the data, we recommend reporting medians, interquartile ranges, and absolute ranges for opioids prescribed and consumed due to the skewed nature of the data. Another limitation of our study is the low number of studies conducted in the early years of this analysis (2004-2008), which may limit characterization of opioid prescription and consumption during this period. In addition, studies differed with regard to the inclusion of preoperative opioid users, and inclusion of potential confounders such as chronic pain or mental health conditions known to influence pain. Most of the included studies examined opioid prescribing practices in academic medical settings, which have been found to prescribe more opioids than non-teaching facilities [36]. The extent to which these findings would generalize to non-academic settings is unclear. Finally, this systematic review did not publish an a priori protocol, which might have addressed any concerns about potential bias in its conclusions. The submission of a public protocol allows for peer review of research methods early in the review process, mitigating the potential effects of author biases, and provides readers a tracking mechanism for changes in the review process [37].

Results of this meta-analysis suggest individual variability in the extent of opioids used and consequently leftover postoperatively. Our review indicates that additional research is needed to identify the sources of this variability at a more granular level and in a manner that might be pragmatically useful in a precision medicine context. Studies of this issue to date consistently report 
only a small number of factors, primarily procedurerelated and demographic, that might drive the extent of opioids left unused postoperatively. Although potentially more challenging pragmatically, it would be valuable to obtain preoperative measures of constructs other literature suggests may influence opioid use. Attitudes towards opioid use are one factor that may influence a patient's actual use of opioids [38]. Negative affect (e.g., depression, anxiety) has also been shown to be predictive of responsiveness to opioids and extent of postoperative opioid use [39-41]. We further note the importance of considering patient-reported pain intensity as a context for interpreting opioid use outcomes. Unfortunately, pain intensity was not reported in some studies, and was inconsistently reported across the other studies (i.e., at differing time intervals, using different rating scales, and addressing differing characteristics-worst, average, current), and therefore could not be examined systematically in our analysis. Recognizing that timing of clinically meaningful measurement of primary endpoints may vary by surgical procedure [42], future work would benefit from consistent inclusion of validated measures of postoperative pain intensity, ideally obtained concurrently with opioid use data. For example, telephone follow-up that assesses both opioid use and pain ratings over the same period of time would enhance interpretation of opioid data. Obtaining these data at a standard time point across studies, such as one-week postdischarge, would also enhance ability to compare opioid data across studies. Clinical practice guidelines developed by an interdisciplinary expert panel recommend that clinicians use a validated pain assessment tool to track responses to postoperative pain treatment [43]. Given likely patient heterogeneity (in terms of cognitive status, education, etc.) in studies of postoperative opioid use, a simple validated pain measure might be optimal. For simplicity and standardization, we recommend a 010 numeric rating scale (NRS) anchored with "no pain" and "worst possible pain" for assessing average pain at rest in the past week (consistent with the suggested follow-up period above). NRS intensity ratings are a preferred outcome in pain trials [44], and retrospective ratings of average pain appear to correspond well with diary-based ratings of momentary pain over the same time period [45]. Measures of pain at rest appear largely to parallel alternative measures of pain relief or pain evoked by activity relevant to the specific surgical procedure [46]. Consistent availability of pain intensity data as described above would facilitate meta-analyses that could evaluate whether the degree of leftover opioids is being driven by differences in actual pain experience, or, alternatively, non-pain factors. For example, evidence in the chronic pain context suggests that opioids may be used not only for pain control, but to reduce negative mood [47]. More comprehensive phenotyping of patients in studies of postoperative opioid consumption going forward could significantly enhance the scientific value of future studies on this topic.

\section{Conclusions}

In summary, results of this meta-analysis of 44 studies reveal that $61 \%$ of opioids prescribed following surgery remain unused, providing a large quantity of opioids potentially available for diversion. There is, however, variability in the amount of opioids leftover, with nonabdominal soft tissue surgeries having the highest proportion of opioids leftover compared to abdominal/pelvic and orthopedic procedures. Less invasive laparoscopic procedures also are associated with a higher proportion of opioids leftover compared to open surgical procedures. The current findings for the first time document and quantify these differences across a wide-range of studies, and underscore the potential problems with regulatory efforts to broadly limit postsurgical opioid prescribing without adequately considering surgical characteristics. Better data to guide such regulatory changes and data-driven physician education regarding optimal procedure-specific opioid prescribing are both needed to achieve the goal of minimizing leftover opioids while continuing to provide adequate pain management in the postoperative period.

\section{Supplementary information}

Supplementary information accompanies this paper at https://doi.org/10. 1186/s13643-020-01393-8.

Additional file 1. Supplemental documentation.

Additional file 2. Effect sizes for mean proportions of opioids leftover.

\section{Abbreviations}

b: Unstandardized regression coefficient; Cl: Confidence interval; CINAHL: Cumulative Index of Nursing and Allied Health Literature; CV: Coefficient of variation; MINORS: Methodological Index for NonRandomized Studies; MME: Morphine milligram equivalents; PRISMA: Preferred Reporting Items for Systematic Review and Meta-Analysis; RVE: Robust variance estimation; SE: Standard error; US: United States

\section{Acknowledgements \\ The authors are grateful to Drs. Elizabeth Tipton and Mark Lipsey for their valuable guidance regarding the data analytic strategy.}

\section{Authors' contributions}

LS, AS, MM, and MD had full access to all of the data in the study and takes responsibility for the integrity of the data and the accuracy of the data analysis. Concept and design: LS, AS, SO, SB. Acquisition, analysis, or interpretation of data: $L S, A S, M M, S O, P W, M D, S B$. Drafting of the manuscript: $L S, M M, S O, P W, M D, S B$. Critical revision of the manuscript for important intellectual content: LS, AS, SO, MD, SB. Statistical analysis: MM, MD. Obtained funding: No unique funding was obtained for this project. Administrative, technical, or material support: N/A. Supervision: MD. The author(s) read and approved the final manuscript. 


\section{Funding}

AS's effort was supported by Oregon Clinical and Translational Research Institute (OCTRI), grant number TL1TR002371 from the National Center for Advancing Translational Sciences (NCATS) and the Vanderbilt Department of Anesthesiology Clinician Scientist Training in Perioperative Science Fellowship (T32GM108554) from the National Institutes of Health. SO's effort was supported through the Building Interdisciplinary Research Careers in Women's Health (2K12HD043483-17). SB's effort was supported by grant number R01DA037891 and R01AG048915 from the National Institutes of Health. The content is solely the responsibility of the authors and does not necessarily represent the official views of the National Institutes of Health.

\section{Availability of data and materials}

The datasets during and/or analyzed during the current study available from the corresponding author on reasonable request.

\section{Ethics approval and consent to participate}

Not applicable

\section{Consent for publication}

Not applicable

\section{Competing interests}

The authors declare that they have no competing interests.

\section{Author details}

${ }^{1}$ School of Nursing, Vanderbilt University, 46121 st Avenue South, Nashville, TN 37240, USA. ²Department of Anesthesiology, Vanderbilt University Medical Center, Nashville, TN, USA. ${ }^{3}$ Department of Psychiatry and Human Behavior, University of Mississippi Medical Center, Jackson, MS, USA ${ }^{4}$ Department of Obstetrics \& Gynecology, Vanderbilt University Medical Center, Nashville, TN, USA. ${ }^{5}$ Eskind Biomedical Library, Vanderbilt University, Nashville, TN, USA. ${ }^{6}$ Department of Biostatistics, Vanderbilt University Medical Center, Nashville, TN, USA

Received: 9 December 2019 Accepted: 20 May 2020

Published online: 11 June 2020

\section{References}

\section{-studies included in meta-analysis}

1. Murphy SL, Xu JQ, Kochanek KD, Anas E. Mortality in the United States, 2017. National Center for Health Statistics: Hyattsville, MD; 2018.

2. Levy B, Paulozzi L, Mack KA, Jones CM. Trends in opioid analgesicprescribing rates by specialty, U.S., 2007-2012. Am J Prev Med. 2015;49(3): 409-13.

3. National Quality Forum. NQF-Endorsed Measures for Surgical Procedures, 2015-2017. Washington, DC; 2018

4. - Fujii MH, Hodges AC, Russell RL, Roensch K, Beynnon B, Ahern TP, et al. Post-discharge opioid prescribing and use after common surgical procedures. J Am Coll Surg. 2018;226(6):1004-12.

5. - Hill MV, McMahon ML, Stucke RS, Barth RJ Jr. Wide variation and excessive dosage of opioid prescriptions for common general surgical procedures. Ann Surg. 2017;265(4):709-14.

6. - Howard R, Fry B, Gunaseelan V, Lee J, Waljee J, Brummett C, et al. Association of opioid prescribing with opioid consumption after surgery in Michigan. JAMA Surg. 2018:e184234.

7. Lipari R. N., Hughes A. How people obtain the prescription pain relievers they misuse. Rockville, MD: Center for Behavioral Health Statistics and Quality, Substance Abuse and Mental Health Services Administration (SAMHSA); 2017.

8. - Bateman BT, Cole NM, Maeda A, Burns SM, Houle TT, Huybrechts KF, et al. Patterns of opioid prescription and use after cesarean delivery. Obstet Gynecol. 2017;130(1):29-35.

9. - Osmundson SS, Raymond BL, Kook BT, Lam L, Thompson EB, Schornack $L A$, et al. Individualized compared with standard postdischarge oxycodone prescribing after cesarean birth: a randomized controlled trial. Obstet Gynecol. 2018;132(3):624-30.

10. Brummett CM, Waljee JF, Goesling J, Moser S, Lin P, Englesbe MJ, et al. New persistent opioid use after minor and major surgical procedures in US adults. JAMA Surg. 2017;152(6):e170504.
11. Sun EC, Darnall BD, Baker LC, Mackey S. Incidence of and risk factors for chronic opioid use among opioid-naive patients in the postoperative period. JAMA Intern Med. 2016;176(9):1286-93.

12. Brat GA, Agniel D, Beam A, Yorkgitis B, Bicket $M$, Homer $M$, et al. Postsurgical prescriptions for opioid naive patients and association with overdose and misuse: retrospective cohort study. Bmj. 2018;360:55790.

13. CO*RE. Opioid Education: State Information Hub 2019. http://core-rems.org/ opioid-education/state-information-hub/. Accessed 12 Dec 2019.

14. Correll D. Chronic postoperative pain: recent findings in understanding and management. F1000Research. 2017;6:1054

15. - Kim N, Matzon JL, Abboudi J, Jones C, Kirkpatrick W, Leinberry CF, et al. A prospective evaluation of opioid utilization after upper-extremity surgical procedures: identifying consumption patterns and determining prescribing guidelines. J Bone Joint Surg Am. 2016;98(20):e89.

16. Bicket MC, Long JJ, Pronovost PJ, Alexander GC, Wu CL. Prescription opioid analgesics commonly unused after surgery: a systematic review. JAMA Surg. 2017:152(11):1066-71.

17. Feinberg AE, Chesney TR, Srikandarajah S, Acuna SA, McLeod RS. Opioid use after discharge in postoperative patients: a systematic review. Ann Surg. 2018;267(6):1056-62.

18. Gerbershagen HJ, Aduckathil S, VanWijck AJ, Peelen LM, Kalkman CJ, Meissner W. Pain intensity on the first day after surgery: a prospective cohort study comparing 179 surgical procedures. Anesthesiology. 2013; 118(4):934-44.

19. Jiang $X$, Orton M, Feng R, Hossain E, Malhotra NR, Zager EL, et al. Chronic opioid usage in surgical patients in a large academic center. Ann Surg. 2017;265(4):722-7.

20. Liberati A, Altman DG, Tetzlaff J, Mulrow C, Gotzsche PC, loannidis JP, et al. The PRISMA statement for reporting systematic reviews and meta-analyses of studies that evaluate health care interventions: explanation and elaboration. J Clin Epidemiol. 2009;62(10):e1-34.

21. Burden M, Keniston A, Wallace MA, Busse JW, Casademont J, Chadaga SR, et al Opioid utilization and perception of pain control in hospitalized patients: a cross-sectional study of 11 sites in 8 countries. J Hosp Med. 2019:14:e1-9.

22. Humphreys K. Avoiding globalisation of the prescription opioid epidemic. Lancet. 2017:390:437-9.

23. Census retions and divisions of the United States. 2020. https://www2. census.gov/geo/pdfs/maps-data/maps/reference/us_regdiv.pdf. Accessed 28 Feb 2020

24. Gammaitoni AR, Fine P, Alvarez N, McPherson ML, Bergmark S. Clinical application of opioid equianalgesic data. Clin J Pain. 2003;19(5):286-97.

25. Slim K, Nini E, Forestier D, Kwiatkowski F, Panis Y, Chipponi J. Methodological index for non-randomized studies (MINORS): development and validation of a new instrument. ANZ J Surg. 2003;73(9):712-6.

26. Hozo SP, Djulbegovic B, Hozo I. Estimating the mean and variance from the median, range, and the size of a sample. BMC Med Res Methodol. 2005;5:13.

27. Lipsey MW. Practical meta-analysis. Wilson DB, editor. Thousand Oaks, Calif: Sage Publications; 2001.

28. Tanner-Smith EE, Tipton E, Polanin JR. Handling complex meta-analytic data structures using robust variance estimates: a tutorial in R. J Dev Life Course Crim. 2016:2:85-112.

29. Fisher Z, Tipton E. Robumeta: robust variance meta-regression; 2014.

30. Pustejovsky JE. clubSandwich: cluster-robust (sandwich) variance estimators with small-sample corrections. 0.0.0.9000 ed 2015.

31. Higgins JP. Thompson SG. Quantifying heterogeneity in a meta-analysis. Stat Med. 2002:21(11):1539-58.

32. Higgins JPT, Thompson SG, Deeks JJ, Altman DG. Measuring inconsistency in meta-analyses. BMJ. 2003;327(7414):557-60.

33. Borenstein M, Higgins JP, Hedges LV, Rothstein HR. Basics of meta-analysis: I(2) is not an absolute measure of heterogeneity. Res Synth Methods. 2017; $8(1): 5-18$

34. State of Tennessee Public Chapter No. 1039, Stat. 53-10-311 (2018).

35. Bruehl S, Apkarian AV, Ballantyne JC, Berger A, Borsook D, Chen WG, et al. Personalized medicine and opioid analgesic prescribing for chronic pain: opportunities and challenges. J Pain. 2013;14(2):103-13.

36. Cron DC, Hwang C, Hu HM, Lee JS, Dupree JM, Syrjamaki JD, et al. A statewide comparison of opioid prescribing in teaching versus nonteaching hospitals. Surgery. 2019:165(4):825-31.

37. Eden J, Levit L, Berg A. Finding what works in health care: standards for systematic reviews. 2011. Institutes of Medicine, National Academies Press, Washington, D.C. 
38. Older CG, Carr EC, Layzell M. Making sense of patients' use of analgesics following day case surgery. J Adv Nurs. 2010;66(3):511-21.

39. Burns JW, Bruehl S, France CR, Schuster E, Orlowska D, Buvanendran A, et al. Psychosocial factors predict opioid analgesia through endogenous opioid function. Pain. 2017;158(3):391-9.

40. De Cosmo G, Congedo E, Lai C, Primeiri P, Dottarelli A, Aceto P. Preoperative psychologic and demographic predictors of pain perception and tramadol consumption using intravenous patient-controlled analgesia. Clin J Pain. 2008;24(5):399-405.

41. Geha H, Nimeskern N, Beziat JL. Patient-controlled analgesia in orthognathic surgery: evaluation of the relationship to anxiety and anxiolytics. Oral Surg Oral Med Oral Pathol Oral Radiol Endod. 2009;108(3):e33-6.

42. Cohen SP, Wallace M, Rauck RL, Stacey BR. Unique aspects of clinical trials of invasive therapies for chronic pain. Pain Rep. 2019;4(3):e687.

43. Chou R, Gordon DB, de Leon-Casasola OA, Rosenberg JM, Bickler S, Brennan $T$, et al. Management of postoperative pain: a clinical practice guideline from the American pain society, the American Society of Regional Anesthesia and Pain Medicine, and the American Society of Anesthesiologists' committee on regional anesthesia, executive committee, and administrative council. J Pain. 2016;17(2):131-57.

44. Dworkin RH, Turk DC, Farrar JT, Haythornthwaite JA, Jensen MP, Katz NP et al. Core outcome measures for chronic pain clinical trials: IMMPACT recommendations. Pain. 2005;113(1):9-19.

45. Jamison RN, Raymond SA, Slawsby EA, McHugo GJ, Baird JC. Pain assessment in patients with low back pain: comparison of weekly recall and momentary electronic data. J Pain. 2006;7(3):1992-199.

46. Gilron I, Carr DB, Desjardins PJ, Kehlet H. Current methods and challenges for acute pain clinical trials. Pain Rep. 2019;4(3):e647.

47. Carpenter RW, Lane SP, Bruehl S, Trull TJ. Concurrent and lagged associations of prescription opioid use with pain and negative affect in the daily lives of chronic pain patients. J Consult Clin Psychol. 2019;87(10):87286.

48. - Alter TH, llyas AM. A prospective randomized study analyzing preoperative opioid counseling in pain management after carpal tunnel release surgery. J Hand Surgery. 2017:42(10):810-5.

49. - As-Sanie S, Till SR, Mowers EL, Lim CS, Skinner BD, Fritsch L, et al. Opioid prescribing patterns, patient use, and postoperative pain after hysterectomy for benign indications. Obstet Gynecol. 2017;130(6):1261-8.

50. - Bates C, Laciak R, Southwick A, Bishoff J. Overprescription of postoperative narcotics: a look at postoperative pain medication delivery, consumption and disposal in urological practice. J Urol. 2011;185(2):551-5.

51. • Beck PR, Nho SJ, Balin J, Badrinath SK, Bush-Joseph CA, Bach BR Jr, et al. Postoperative pain management after anterior cruciate ligament reconstruction. J Knee Surg. 2004;17(1):18-23.

52. - Cabo J, Hsi RS, Scarpato KR. Postoperative opiate use in urological patients: a quality improvement study aimed at improving opiate disposal practices. J Urol. 2019:201(2):371-6

53. - Chapman T, Kim N, Maltenfort M, llyas AM. Prospective evaluation of opioid consumption following carpal tunnel release surgery. Hand (New York, NY). 2017;12(1):39-42.

54. - Cunningham D, Lewis B, Hutyra C, Nho S, Olson S, Mather R. Prospective, observational study of opioid use after hip arthroscopy for femoroacetabular impingement syndrome. Arthroscopy. 2018;34(5):1488-97 e6.

55. - Griffith K, Clark N, Wright K, Zuckerman A, Ferzandi T. Opioid prescription and patient use following gynecologic procedures. Obstet Gynecol. 2017;129:47S-8S.

56. - Gupta A, Kumar K, Roberts MM, Sanders AE, Jones MT, Levine DS, et al. Pain management after outpatient foot and ankle surgery. Foot Ankle Int. 2018;39(2): 149-54

57. - Harris K, Curtis J, Larsen B, Calder S, Duffy K, Bowen G, et al. Opioid pain medication use after dermatologic surgery: a prospective observational study of 212 dermatologic surgery patients. JAMA Dermatol. 2013;149(3):317-21.

58. - Hart AM, Broecker JS, Kao L, Losken A. Opioid use following outpatient breast surgery: are physicians part of the problem? Plast Reconstr Surg. 2018;142(3):611-20.

59. - Hartford LB, Van Koughnett JAM, Murphy PB, Vogt KN, Hilsden RJ, Clarke CF, et al. Standardization of outpatient procedure (STOP) narcotics: a prospective non-inferiority study to reduce opioid use in outpatient general surgical procedures. J Am Coll Surg. 2019;228(1):81-8 e1.

60. · Hill MV, Stucke RS, Billmeier SE, Kelly JL, Barth RJ Jr. Guideline for discharge opioid prescriptions after inpatient general surgical procedures. J Am Coll Surg. 2018;226(6):996-1003.
61. - Hota LS, Warda HA, Haviland MJ, Searle FM, Hacker MR. Opioid use following gynecologic and pelvic reconstructive surgery. Int Urogynecol J. 2018;29(10):1441-5.

62. - Howard R, Waljee J, Brummett C, Englesbe M, Lee J. Reduction in opioid prescribing through evidence-based prescribing guidelines. JAMA Surg. 2018;153(3):285-7.

63. - llyas AM, Miller AJ, Graham JG, Matzon JL. Pain management after carpal tunnel release surgery: a prospective randomized double-blinded trial comparing acetaminophen, ibuprofen, and oxycodone. J Hand Surg Am. 2018;43(10):913-9.

64. - Kumar K, Gulotta LV, Dines JS, Allen AA, Cheng J, Fields KG, et al. Unused opioid pills after outpatient shoulder surgeries given current perioperative prescribing habits. Am J Sports Med. 2017;45(3):636-41.

65. - Maughan BC, Hersh EV, Shofer FS, Wanner KJ, Archer E, Carrasco LR, et al. Unused opioid analgesics and drug disposal following outpatient dental surgery: a randomized controlled trial. Drug Alcohol Depend. 2016;168:328-34

66. - Merrill HM, Dean DM, Mottla JL, Neufeld SK, Cuttica DJ, Buchanan MM. Opioid consumption following foot and ankle surgery. Foot Ankle Int. 2018; 39(6):649-56.

67. - Miller A, Kim N, Zmistowski B, Ilyas AM, Matzon JL. Postoperative pain management following carpal tunnel release: a prospective cohort evaluation. Hand (New York, NY). 2017;12(6):541-5.

68. - Osmundson SS, Grasch JL, Schornack LA, Young JL, Lisa ZC, Kelly BA, et al. Opioid use after cesarean delivery following hospital discharge. Am J Obstet Gynecol. 2017;216(1 Supplement 1):S411-S2

69. - Patel HD, Srivastava A, Patel ND, Faisal FA, Ludwig W, Joice GA, et al. A prospective cohort study of postdischarge opioid practices after radical prostatectomy: the ORIOLES initiative. Eur Urol. 2019;75(2):215-8.

70. Patel S, Sturm A, Bobian M, Svider PF, Zuliani G, Kridel R. Opioid use by patients after rhinoplasty. JAMA Facial Plastic Surg. 2018;20(1):24-30.

71. - Peters B, Izadpanah A, Islur A. Analgesic consumption following outpatient carpal tunnel release. J Hand Surg Am. 2018;43(2):189 e1-5.

72. - Riley CA, Kim M, Sclafani AP, Kallush A, Kjaer K, Kacker AS, et al. Opioid analgesic use and patient-reported pain outcomes after rhinologic surgery. Int Forum Allergy Rhinol. 2019;9(4):339-44.

73. - Rodgers J, Cunningham K, Fitzgerald K, Finnerty E. Opioid consumption following outpatient upper extremity surgery. J Hand Surg Am. 2012;37(4): 645-50.

74. - Sabatino MJ, Kunkel ST, Ramkumar DB, Keeney BJ, Jevsevar DS. Excess opioid medication and variation in prescribing patterns following common orthopaedic procedures. J Bone Joint Surg Am. 2018;100(3):180-8.

75. - Saini S, McDonald EL, Shakked R, Nicholson K, Rogero R, Chapter M, et al. Prospective evaluation of utilization patterns and prescribing guidelines of opioid consumption following orthopedic foot and ankle surgery. Foot Ankle Int. 2018;39(11):1257-65.

76. - Schmidt P, Berger MB, Day L, Swenson CW. Home opioid use following cesarean delivery: how many opioid tablets should obstetricians prescribe? J Obstet Gynaecol Res. 2018;44(4):723-9.

77. - Solouki S, Plummer M, Agalliu I, Abraham N. Opioid prescribing practices and medication use following urogynecological surgery. Neurourology and Urodynamics. 2018.

78. - Stepan JG, London DA, Osei DA, Boyer MI, Dardas AZ, Calfee RP. Perioperative celecoxib and postoperative opioid use in hand surgery: a prospective cohort study. J Hand Surg Am. 2018;43(4):346-53.

79. - Swarup A, Mathis KA, Hill MV, Ivatury SJ. Patterns of opioid use and prescribing for outpatient anorectal operations. J Surg Res. 2018;229:283-7.

80. - Swenson CW, Kelley AS, Fenner DE, Berger MB. Outpatient narcotic use after minimally invasive urogynecologic surgery. Female Pelvic Med Reconstr Surg. 2016;22(5):377-81.

81. - Tan WH, Yu J, Feaman S, McAllister JM, Kahan LG, Quasebarth MA, et al. Opioid medication use in the surgical patient: an assessment of prescribing patterns and use. J Am Coll Surg. 2018;227(2):203-11.

82. - Tharakan T, Jiang S, Fastenberg J, Ow TJ, Schiff B, Smith RV, et al. Postoperative pain control and opioid usage patterns among patients undergoing thyroidectomy and parathyroidectomy. Otolaryngol Head Neck Surg. 2018;160(3):394-401.

83. - Thiels CA, Ubl DS, Yost KJ, Dowdy SC, Mabry TM, Gazelka HM, et al. Results of a prospective, multicenter initiative aimed at developing opioidprescribing guidelines after surgery. Ann Surg. 2018;268(3):457-68. 
84. Weiland BM, Wach AG, Kanar BP, Castele MT, Sosovicka MF, Cooke MR, et al. Use of opioid pain relievers following extraction of third molars. Compend Contin Educ Dent (Jamesburg, NJ: 1995). 2015;36(2):107-11.

85. • Wojahn RD, Bogunovic L, Brophy RH, Wright RW, Matava MJ, Green JR, et al. Opioid consumption after knee arthroscopy. J Bone Joint Surg (Am Vol). 2018;100(19):1629-36.

\section{Publisher's Note}

Springer Nature remains neutral with regard to jurisdictional claims in published maps and institutional affiliations.

Ready to submit your research? Choose BMC and benefit from:

- fast, convenient online submission

- thorough peer review by experienced researchers in your field

- rapid publication on acceptance

- support for research data, including large and complex data types

- gold Open Access which fosters wider collaboration and increased citations

- maximum visibility for your research: over $100 \mathrm{M}$ website views per year

At BMC, research is always in progress.

Learn more biomedcentral.com/submissions 